\title{
Exploring functional variation affecting ceRNA regulation in humans
}

Mulin Jun $\mathrm{Li}^{1,2}$, Jiexing $\mathrm{Wu}^{1}$, Peng Jiang ${ }^{3}$, Wei $\mathrm{Li}^{3}$, Yun $\mathrm{hh}^{2}$, Daniel Fernandez ${ }^{1}$, Russell J. H. Ryan $^{4,5}$, Yiwen Chen ${ }^{6}$, Junwen Wang ${ }^{2, *}$, Jun S. Liu, ${ }^{1, *}$, X. Shirley Liu ${ }^{3,{ }^{*}}$

${ }^{1}$ Department of Statistics, Harvard University, Cambridge, Boston, Massachusetts, USA.

${ }^{2}$ Department of Biochemistry and Centre for Genomic Sciences, LKS Faculty of Medicine, The University of Hong Kong, Hong Kong SAR, China.

${ }^{3}$ Department of Biostatistics and Computational Biology, Dana-Farber Cancer Institute, Harvard School of Public Health, Boston, Massachusetts, USA.

${ }^{4}$ Broad Institute of MIT and Harvard, Cambridge, Massachusetts, USA.

${ }^{5}$ Department of Pathology, Massachusetts General Hospital and Harvard Medical School, Boston, Massachusetts, USA.

${ }^{6}$ Department of Bioinformatics and Computational Biology, Division of Quantitative Sciences, The University of Texas MD Anderson Cancer Center, Houston, Texas, USA.

*These authors jointly supervised this work

Correspondence and requests for materials should be addressed to Mulin Jun $\mathrm{Li}$ (mulin0424.li@gmail.com) or X. Shirley Liu (xliu@jimmy.harvard.edu)

\section{Abstract}

MicroRNA (miRNA) sponges have been shown to function as competing endogenous RNAs (ceRNAs) to regulate the expression of other miRNA targets in the network by sequestering available miRNAs. As the first systematic investigation of the genome-wide genetic effect on ceRNA regulation, we applied multivariate response regression and identified widespread genetic variations that are associated with ceRNA competition using 462 Geuvadis RNA-seq data in multiple human populations. We showed that SNPs in gene 3'UTRs at the miRNA seed binding regions can simultaneously regulate gene expression changes in both cis and trans by 
the ceRNA mechanism. We termed these loci as endogenous miRNA sponge expression quantitative trait loci or "emsQTLs", and found that a large number of them were unexplored in conventional eQTL mapping. We identified many emsQTLs are undergoing recent positive selection in different human populations. Using GWAS results, we found that emsQTLs are significantly enriched in traits/diseases associated loci. Functional prediction and prioritization extend our understanding on causality of emsQTL allele in disease pathways. We illustrated that emsQTL can synchronously regulate the expression of tumor suppressor and oncogene through ceRNA competition in angiogenesis. Together these results provide a distinct catalog and characterization of functional noncoding regulatory variants that control ceRNA crosstalk.

\section{Introduction}

Recent RNA biology has revealed that specific RNAs can operate as ceRNAs to titrate away the pools of active miRNAs, indirectly regulating the expression of other transcripts targeted by the same set of miRNAs. Endogenous miRNA sponges, including mRNA, pseudogene transcripts, long non-coding RNAs and circular RNAs, have been discovered in quick succession ${ }^{1 ; 2}$, and play critical roles in cellular metabolism and disease development ${ }^{3 ; 4}$. Besides the different type of transcripts, it has been proposed that structure variations can perturb ceRNA competition and initiate subsequent disease pathway, such as large deletion and insertion, copy number variation, as well as chromosomal translocation ${ }^{5-7}$. ceRNA crosstalk is determined by miRNA response elements (MRE), which encode the ceRNA regulatory network and sustain the dynamic equilibrium for all ceRNAs and miRNAs within the network ${ }^{8}$. Under this circumstance, any genetic event affecting MRE will trigger the perturbation of ceRNA regulation by titrating miRNA availability. One example is SNP rs17228616, which disrupts the interaction between miR-608 and AChE and suppresses other miR-608 targets such as CDC42 and IL6 ${ }^{9}$. 
Nevertheless, there has been no study to our knowledge that systematically tests whether such genetic effect is pervasive in the human evolution and populations.

Quantitative traits, such as gene expression and epigenetic modifications, are thought to be largely heritable during species evolution ${ }^{10 ;}{ }^{11}$. Recently next generation sequencing technologies have enabled us to unravel the correlation between genetic variants and different molecular phenotypes, and facilitated the discovery of many human quantitative trait loci $(\mathrm{QTLs})^{12}$. For miRNA-related molecular traits, researchers have discovered many miRNA gene expression QTLs (miRNA-eQTL) that control miRNA gene expression ${ }^{13-16}$ and $3^{\prime}$ untranslated region (3'UTR)-eQTLs that are associated with miRNA target expression ${ }^{17}$; . With a better knowledge of ceRNA crosstalk and competition, it is very important to understand how genetic polymorphisms shape human ceRNA regulation. Using 1000 Genomes Project genotype and Geuvadis RNA-seq phenotype data, our study here represents the first effort to investigate the genetic variants associated with ceRNA crosstalk.

\section{Material and Methods}

\section{The logic for detecting genetic variants affecting ceRNA regulation}

We assume that genetic variants, such as SNPs and indels that affect miRNA response elements (MREs) will perturb ceRNA regulation by titrating miRNA availability. Specifically, a causal variation in the seed region of miRNA binding site can introduce different consequences of miRNA-target interaction, by creating or erasing an MRE, or strengthening or weakening an MRE in a ceRNA driver gene (ceD). These subtle changes could perturb the original miRNA and ceRNA regulatory network and the dynamic distribution of other miRNA target genes. Since we focused on the independent effect of one SNP or indel in one MRE of a ceD, the variants will 
mostly affect the distribution of associated miRNA and its direct targets (ceTs) although the cascade effect could impact other miRNAs and ceRNAs (Supplemental Figure S1). To simplify the investigation of variant effect, we focused on unique miRNA-centered regulatory network in this study. A SNP or indel that creates or strengthens an MRE will decrease the expression of its own host gene (refer to ceD in this study) and increase the expression of the corresponding ceT, while a SNP or indel that erases or weakens an MRE will have the opposite effect. We therefore termed these variants candidate endogenous miRNA sponge (ceRNA) expression quantitative trait loci (emsQTLs).

\section{Expression data and genotype data}

We used Geuvadis RNA sequencing data and small RNA sequencing data of 462 unrelated human lymphoblastoid cell line samples from the CEPH (CEU), Finns (FIN), British (GBR), Toscani (TSI) and Yoruba (YRI) populations in 1000 Genomes project ${ }^{16 ; 19}$. To be consistent with Geuvadis eQTL detection framework, we directly utilized Geuvadis quantifications for gene and miRNA expression. Geuvadis also provides 462 human genotypes as processed VCFs, of which 421 samples are from 1000 Genomes project Phase1 release v3 and 42 samples are from 1000 Genomes project Phase 2 Omni 2.5M genotype array with imputation. We used major allele frequency (MAF) > 5\% as cutoff to select variants for downstream analysis.

\section{Construct variant-miRNA-ceD-ceT unit}

We extracted human 3'UTR sequences according to GENCODE ${ }^{20}$ V12 annotation (consistent with Geuvadis RNA-seq quantification) and 714 Geuvadis quantified miRNA sequences from miRBase $^{21}$. We mapped Geuvadis biallelic genotypes to 3'UTR sequences to construct reference and mutant miRNA targets. Assuming variant independence, we used TargetScan 6.2 to predict miRNA-target relationship ${ }^{22}$. To select reliable miRNA-target pairs, we filtered the prediction by TargetScan Context+ Score (TargetScan cutoff: $<-0.310$ for 8 mer, $<-0.161$ for 
7mer-m8, < -0.099 for 7 mer-1A). We also adopted ViennaRNA Package ${ }^{23}$ to estimate the secondary structure-based energies of mRNA with $\left(\Delta G_{\text {duplex }}\right)$ or without $\left(\Delta G_{\text {open }}\right)$ interaction with miRNA, and used RNAhybrid to display the binding pattern²3. We then selected those "variantmiRNA-target" units that meet any of the following three criteria: 1) target gain: sequence with alternative allele is a miRNA target site but not for reference allele; 2) target loss: reference allele is but alternative allele is not a miRNA target site; 3) change of context+ score between target sites with reference allele and alternative allele. We treated the miRNA targets meeting the above criteria as putative ceDs. For each selected variant-miRNA-ceD unit, we further searched candidate ceTs under the control of the same miRNA as the ceD (on either reference and mutant 3'UTR) according to TargetScan prediction. Finally, we tested the association between a genetic variant and each ceD-ceT pair under a miRNA-centered regulatory network.

\section{Control for confounding factors}

The quantifications of gene and miRNA expression are usually affected by different technical variations and hidden factors, which will reduce power to interpret expression variability caused by genetic factors. To maximize the emsQTL detection power, we used PEER ${ }^{24}$ to estimate the hidden confounding factors $(K)$ in expression quantifications, and selected the first ten PEER factors $(K=10)$ according to the performance report of Geuvadis eQTL detection. We also calculated expression residuals for miRNA quantifications after accounting for estimated PEER factors, which serve as an essential confounder to control miRNA expression variability. In order to control population stratification, we performed principal component analysis to estimate principal components (PCs) for 462 individual genotype data and selected first three PCs as additional model covariates in the QTL analyses.

\section{Multivariate linear model}


We wanted to test if the variant regulates the expression of ceD and ceT in a reciprocal pattern due to the ceRNA competition. This can normally be achieved by a two-step linear regression on gene expressions against genotype and a set of confounders, with ceD selection in the first step and ceT selection in the second step. However, expressions of the ceD and ceT pairs usually show positive correlation at the functional allele state, suggesting that we could benefit from modeling the relationship between these two responses jointly. Therefore, we used the multivariate linear regression on two responses to simultaneously model the genetic contribution on variability of ceRNA regulation. For each variant-miRNA-ceD-ceT unit, we considered gene expression, measured as the sum of all transcript RPKMs of ceD $\left(Y_{d}\right)$ and ceT $\left(Y_{t}\right)$, as two dependent variables and transform them to standard normal. We further incorporated the following confounding factors beside the individual genotype $(G)$ : the PEER residual of miRNA expression $\left(M_{r}\right)$, the 10 PEER factors of ceD expression $\left(P F_{d}\right)$, the 10 PEER factors of ceT expression $\left(P F_{t}\right)$, and first three PCs of individual genotype $(P C)$. The separate regression model is shown below:

$Y_{d}=\beta_{01}+\beta_{11} G+\beta_{21} M_{r}+\beta_{31} P F_{d}+\beta_{41} P F_{t}+\beta_{51} P C+\varepsilon_{1}$

$Y_{t}=\beta_{02}+\beta_{12} G+\beta_{22} M_{r}+\beta_{32} P F_{d}+\beta_{42} P F_{t}+\beta_{52} P C+\varepsilon_{2}$

The multivariate regression on both ceD and ceT:

$\left(Y_{d}, Y_{t}\right)=G+M_{r}+P F_{d}+P F_{t}+P C+\varepsilon$

where we simultaneously measured two responses $Y_{d}$ and $Y_{t}$, and the same set of predictors on each sample unit. $\varepsilon=\left(\varepsilon_{1}, \varepsilon_{2}\right)^{\prime}$ has expectation zero and an unknown covariance matrix. The errors associated with different responses on the same sample unit may have different variances and may be correlated. 
Thus, the variant effect for ceD and ceT can be estimated by the corresponding coefficients on genotype $\left(\beta_{d}\right.$ and $\left.\beta_{t}\right)$, and we measured this multivariate model by Pillai's trace test statistics. However, in our definition of emsQTLs, we required opposite expression trend between ceD and each of its ceTs accompanying the genotype change $(A A=0, A B=1$ and $B B=2)$, so we further filtered variants to only keep those with their $\beta_{d}$ and $\beta_{t}$ values having opposite signs $\left(\beta_{d} \times \beta_{t}<0\right)$. We finally reported the variant-miRNA-ceD-ceT unit with Benjamini-Hochberg false discovery rate $<0.05$.

\section{emsQTL attributes analysis}

To be consistent with the Geuvadis RNA-seq quantification, we utilized GENCODE V12 gene annotation to investigate the functional properties of ceDs and ceTs. We also used DAVID to find enriched gene annotations and pathways ${ }^{25}$. We used SNVrap to annotate genetic variants ${ }^{26 ;}$ 27, and grouped the GWAS traits according to ontology mapping (human phenotype ontology and disease ontology) of GWASdb 28 .

\section{Evolutionary analysis}

We used six statistical measurements, including difference of derived allele frequency (DDAF) ${ }^{29}$, fixation Index $\left(F_{\mathrm{ST}}\right)^{30}$, Tajima's D (TD $)^{31}$, integrated haplotype score (iHS) ${ }^{32}$, cross-population extended haplotype homozygosity $(\mathrm{XPEHH})^{33}$ and cross-population composite likelihood ratio $(\mathrm{XPCLR})^{34}$, to evaluate signals of positive selection on each detected emsQTL SNP using genotype data from five populations of 1000 Genomes project (CEU, FIN, GBR, TSI and YRI). Statistical significance was evaluated by dbPSHP ${ }^{35}$, and we only kept emsQTL SNP with at least one statistically significant score out of the six scores (Supplemental Table S1). Hierarchical clustering was used to cluster the selected emsQTLs according to their derived allele frequencies (DAF).

\section{Functional prediction of variant effect}


We adopted TargetScan context+ score and combined interaction energy $\operatorname{score}^{36}(\Delta \Delta \mathrm{G}=$ $\left.\Delta \mathrm{G}_{\text {duplex }}-\Delta \mathrm{G}_{\mathrm{open}}\right)$ to measure the alteration of binding affinity for each miRNA-target interaction. We then calculated the distance of context+ score ( $\Delta$ context+ score) and the distance of combined interaction energy $(\Delta \Delta \Delta \mathrm{G})$ between alternative allele and reference allele, which are used to predict the miRNA-target binding affinity change. In analogy with $\beta_{d}$, a negative score represents the gain-of-function effect, whereas a positive score represents the loss-of-function effect.

\section{GWAS enrichment}

GWAS traits/diseases associated SNPs (TASs) were collected from GWASdb, NHGRI GWAS Catalog ${ }^{34}, \mathrm{HuGE}^{37}$, PheGenl ${ }^{38}$ and GRASP ${ }^{35}$, resulting in 33,645 significant SNPs with $P<1 \mathrm{E}-5$. To link the signal in the linkage disequilibrium (LD) region, we calculated SNP correlations by MATCH based on the 1000 Genomes project super population for EUR. We obtained all linked SNPs with $r^{2}>0.8$ for each GWAS leading TAS and identified emsQTLs overlapping with this expanded list. To test the enrichment of emsQTLs in GWAS signals, we prepared two background datasets for the SNP distribution in the miRNA binding site. We mapped all 1000 Genomes project SNPs into miRNA seed binding region predicted by TargetScan as the first background. We further required that SNPs in first background should have changed binding affinity ( $\Delta$ context+ score is not equal to zero) under different alleles to form the second strict background. Using those two backgrounds, we overlapped them with extended GWAS signals and tested the enrichment by hypergeometric test.

\section{Results}

\section{Genetic Effects on ceRNA Regulation in Human Populations}


Our variant selection pipeline (Supplemental Figure S2) successfully mapped 3,544 unique genetic variants (including 3,263 SNPs and 281 indels) on miRNA seed binding sites in the 3'UTR of 2,753 genes (putative ceDs) using 462 Geuvadis individuals. These loci have shown differentiated interaction patterns with 439 miRNAs (out of 714 profiled by Geuvadis) between the reference and alternative alleles. For each putative ceD, we can match over hundreds of other genes (ceTs) which are targeted by the same miRNA according to TargetScan prediction. We applied the multivariate linear regression (in Equation 3) to detect genomic loci that regulate expression levels of a ceD and each of the corresponding ceT. The model includes several essential confounding factors as regressors, including miRNA expression for controlling variability of miRNA concentration among individuals, PEER factors $(\mathrm{K})$ estimating the hidden confounding factors of RNA-seq quantifications, and principal components of individual genotype accounting for population stratification. This multivariate linear model can simultaneously test for two responses of both ceD and ceT expressions and take advantage of the potentially correlated nature between ceD and ceT. After controlling at the FDR of $5 \%$, we further filtered out units with $\beta_{d} \times \beta_{t}>0$ and only retained those showing opposite signs of association between genetic variants and gene expression of the two ceRNAs.

\section{Genome-wide detection of emsQTLs in different populations}

We applied the model and the filtering strategy to five Geuvadis populations independently and successfully detected many emsQTLs. We found 67 (CEU, 91 individuals), 97 (FIN, 95 individuals), 106 (GBR, 94 individuals), 66 (TSI, 93 individuals) and 47 (YRI, 89 individuals) significant associations of unique variant at 5\% FDR respectively (Supplemental Table S2). To improve the detection power, we merged the four European subpopulations (EUR, 373 individuals) and detected 387 total significant emsQTLs and 1,875 variant-miRNA-ceD-ceT units (Supplemental Table S3, Figure 1). In the 387 emsQTLs associated with the EUR population, 344 are SNPs and remainings are indels (Figure 2A), suggesting an enrichment of indels over 
SNPs in affecting miRNA-target interaction and subsequent ceRNA regulation $(P=0.04$, chisquare test). Different from eQTL detection, emsQTL are not only directly associated with its located ceD in cis, but also associated with the corresponding ceT in trans through their common miRNA regulator. To investigate if some functional eQTLs can be explained by ceRNA regulation, we checked the number of emsQTLs that overlap with the Geuvadis fine mapping ("the best") eQTL result from the EUR population, and found to our surprise only 6 overlaps. However, when we considered the Geuvadis all mapped eQTLs, the overlap of emsQTLs is significantly improved (43\%, Figure 2B). This suggests that other independent associations may exist in the linked region of each finely mapped eQTL, and the emsQTLs spectrum have pinpointed many additional associations that were missed by conventional eQTL analyses.

\section{Positive selection on ceRNA regulation}

By simply overlapping the emsQTLs in different subpopulations, we can also find many loci in common or specific to each population (Figure 2C and 2D). Similar patterns can be observed for related miRNAs, ceDs and ceTs of emsQTLs as well (Supplemental Figure S3). Surprisingly, the number of detected emsQTLs is drastically different among different subpopulations despite their similar sample sizes and expected statistical power. When we ranked the number of detected emsQTLs of each subpopulation in ascending order, we found that the sequence of subpopulations (YRI, TSI, CEU, FIN, GBR) follows precisely the human migration path in Europe (Supplemental Figure S4). This phenomenon may suggest that the recent positive selection is shaping the evolution of ceRNA regulation in human populations due to migration and subsequent adaption. To investigate whether emsQTLs are putative targets of the recent positive selection, we screened emsQTLs using six statistical measures (DDAF, $F_{\mathrm{ST}}, \mathrm{TD}$, iHS, XPEHH, and XPCLR) for each subpopulation. We found 46 emsQTLs with positively selected signals for at least one of the measures according to their corresponding empirical thresholds (Supplemental Table S4). Hierarchical clustering for derived allele frequencies of these 46 
genetic variants clearly recovers the population relationship and shows distinct pattern on individual locus (Figure 3A). For example, one of YRI-specific emsQTLs rs1050286 $(P=8.11 \mathrm{E}-$ 6) shows disparate derived allele frequency between African (DAF of YRI: 0.89) and European population (DAF of TSI: 0.44; CEU: 0.47; GBR: 0.54; FIN: 0.53) (Supplemental Figure S5). SNP rs1050286 has four measures passing the significant cutoff in the YRI population (DDAF: 0.266; $F_{\text {ST: }}$ 0.12; iHS: 2.285; XPEHH: 1.24), suggesting its likely positive selection in YRI. Long-range haplotype analysis also confirmed the selective sweep around this locus (Figure 3B and 3C). This population-specific emsQTL was detected by our model to regulate ceD OLR1 and ceT HORMAD2 by miR-149-5p, and derived allele $A$ enhances the binding affinity for miR-149-5p and OLR1 according to direction of $\beta_{d}$ coefficient (positive value). Therefore, the gene expression of $O L R 1$ is down-regulated in allele A state, which increases the gene expression of target ceRNA HORMAD2 from miRNA sponge effect. Previous studies have implicated the overexpression of OLR1 gene in many diseases including alzheimer's disease, atherosclerosis, myocardial infarction, obesity, dyslipidemia, and cancer ${ }^{39-41}$. Our ceRNA analysis suggests that the protective role of allele A on rs1050286 in African population arises from miR-149-5p shifting its targets from HORMAD2 to OLR1 and reduces the OLR1 expression through ceRNA competition.

\section{Putative Causality of emsQTLs}

\section{Evaluating emsQTLs properties with functional investigations}

Functional interpretation of emsQTLs is pivotal to our understanding their underlying biological mechanisms and phenotype causality. Coefficients of ceD and ceT in our regression model can reflect the degree of gene expression perturbation under different genotypes. Using the EUR 387 emsQTLs, we found a majority of $\beta_{d}$ and $\beta_{t}$ to be small $(<1)$ in the 1,875 significant variant- 
miRNA-ceD-ceT units (Figure 4A and 4B), which indicates a moderate effect of these genetic variations in increasing the precision of target gene expression and ceRNA regulation. To investigate whether the 298 emsQTL-associated ceDs and 1,459 ceTs are engaged in important biological processes, we performed DAVID gene-annotation enrichment analysis for these two gene sets. ceDs were enriched in nucleotides binding, suppressor of cytokine signaling family protein binding and DNA repair (Supplemental Figure S6), while ceTs were enriched in sialyltransferase function, tyrosine protein kinase function, positive gene regulation, transcription regulation and cell proliferation (Supplemental Figure S7). These may indicate that many emsQTLs affect expressions of transcriptional regulators and signaling genes directly, and then regulate expressions of other genes through the ceRNA competition mechanism.

The variant's effect on miRNA-target interaction can be assessed by functional prediction algorithms that have been developed to estimate the change of binding affinity among different variant alleles $26 ; 42 ; 43$. To evaluate whether the direction of association $\left(\beta_{d}\right)$ is concordant with computational prediction on ceD through variant effect in cis, we calculated two scores, $\Delta$ context+ score and $\Delta \Delta \Delta \mathrm{G}$, using TargetScan and an energy-based method ${ }^{36}$ for the 387 emsQTLs in EUR population. Intuitively, $\Delta$ context+ score reflects the discrepancy of binding affinity and $\Delta \Delta \Delta G$ measures difference of combined interaction energy between alternative allele and reference allele. We found $53 \%$ and $39 \%$ of emsQTLs have $\beta_{d}$ in consistent direction with $\Delta$ context+ score and $\Delta \Delta \Delta \mathrm{G}$ in functional prediction respectively (Figure $4 \mathrm{C}$ ), and this increases to $75 \%$ if we consider the consistency with either $\Delta$ context+ score or $\Delta \Delta \Delta G$ as independent validation (Figure $4 \mathrm{C}$ ). These results suggest that majority of detected emsQTLs can be validated by functional prediction in their ceD locus.

The concordance of direction between statistical association and functional prediction can help us in silico prioritize the emsQTL candidates with potential causal evidence. From the 1,413 concordant variant-miRNA-ceD-ceT units for 250 emsQTLs, one can infer their molecular 
causality from both computational prediction and quantitative interpretation. Here, we use an example to illustrate how the emsQTL works. SNP rs1056984 is predicted to affect the seed binding between hsa-miR-296-5p and 3'UTR of DIDO1. TargetScan predicted binding under ancestral allele G (7mer-m8, context+ score: -0.236$)$, but not under the derived allele $A$ on this SNP. Further thermodynamic estimation confirmed that allele $\mathrm{G}$ has better binding affinity to miRNA (MFE of A allele: $-26.7 \mathrm{kcal} / \mathrm{mol}$; MFE of allele $\mathrm{G}:-30.5 \mathrm{kcal} / \mathrm{mol}$ ). The simulated binding pattern also shows that allele $G$ will enhance the binding stability by creating $G: C$ match to position 8 of hsa-miR-296-5p (Figure 5A and 5B). Our model detected rs1056984 to be an emsQTL $(P=4.76 \mathrm{E}-05)$ that controls the regulation among hsa-miR-296-5p, ENSG00000101191 (DIDO1) and ENSG00000185361 (TNFAIP8L1). The $\beta_{d}$ value of ceD DIDO1 is -1.34 (Figure 5C), and the $\beta_{t}$ value of ceT TNFAIP8L1 is 0.20 (Figure 5D). The negative value of $\beta_{d}$ further shows that rs1056984 is perhaps a gain-of-function mutation, which is consistent with functional prediction. This reversed relationship between coefficients indicates that genetic effect is driving the competing process of ceRNAs regulation. As the sequence of this allele changes from AA to AG to GG, the gradually enhanced sponge effect down-regulates DIDO1 expression (ceD) and up-regulates TNFAIP8L1 expression (ceT). When this locus is homozygous GG, we observed a significantly positive correlation (Cor $=0.29, P=0.01$ ) between DIDO1 and TNFAIP8L1, further supporting the interaction between ceD and ceT through competition for hsa-miR-296-5p (Figure 5E).

When we searched biological functions for DIDO1, TNFAIP8L1 and miR-296-5p, we found that this emsQTL may represent a new mechanism for miR-296-5p triggered carcinogenesis. miR296 has been characterized as "angiomiR" which can regulate angiogenesis ${ }^{44}$. It is reported to have a specific role to promote tumor angiogenesis by targeting HGS mRNA and resulting in the overexpression of VEGF receptors in angiogenic endothelial cells $s^{45-47}$. MiR-296 may also contribute to carcinogenesis by dysregulating $\mathrm{p} 53^{48}$. In this scenario, DIDO1 gene is a tyrosine- 
phosphorylated putative transcription factor, previously thought to induce apoptosis and mitotic division ${ }^{49}$ 50, and might be a tumor suppressor gene ${ }^{51}$. In contrast, majority of publications reported TNFAIP8L1 to be an antiapoptotic molecule and oncogene in developing many cancers $^{13 ;}$ 52-54. Here, we predicted emsQTL rs1056984 to affect the ceRNA regulation in switching the expression of tumor suppressor and oncogene under different genotypes. Specifically, efficient miRNA competition occurs in the ancestral allele G, however, the derived allele A of rs1056984 has a protective effect in maintaining tumor suppressor DIDO1 expression and inhibiting oncogenic TNFAIP8L1 expression by shifting miR-296-5p binding from DIDO1 to TNFAIP8L1 (Figure 5F). Although there is no diseases/traits associated evidences for rs1056984 at the current stage, we found that African population have lower derived allele frequency (DAF of $\mathrm{YRI}$ is 0.28 , DAF of $\mathrm{CEU}$ is 0.65 ) in the 1000 Genomes project (Supplemental Figure S8). Further calculation on $F_{\mathrm{ST}}(0.24)$ between CEU and YRI indicates that positive selection may drive the evolution of this locus.

emsQTLs explain GWAS traits and diseases associated signals in miRNA binding sites

To investigate if emsQTL-affected gene expression changes contribute to human phenotypes, we connected emsQTLs in EUR population to GWAS trait/disease-associated SNPS (TASs), and found 8 of 387 ubiquitous emsQTLs to overlap with GWAS leading TASs (Table 1). The top mapped TAS rs7294, which locates in the 3'UTR region of VKORC1, has been frequently shown to be associated with warfarin maintenance dose in anticoagulant therapy ${ }^{55-57}$. Individuals with derived allele A produce less Vitamin $\mathrm{K}$ epoxide reductase than those with the G allele ("non-A haplotype"), thus the former need lower warfarin doses to inhibit the enzyme and produce an anticoagulant effect ${ }^{58}$. Our emsQTL analysis suggests that at the molecular level, allele A of rs7294 may promote the interaction among miR-147a and VKORC1 target site $\left(\beta_{d}=-1.12\right)$ to down-regulate $V K O R C 1$ expression (Supplemental Figure S9A), consistent with 
the previously observed VKORC1 expression in A allele individuals ${ }^{58}$. Also, the competition effect of this locus can help connect VKORC1 to three significant ceTs EIF2B5, LRRFIP1 and RPTOR (Supplemental Figure S9B-D) that are important in translational initiation or signaling pathway regulation.

Since GWAS TAS may not be causal, we further scanned SNPs in high LD $\left(r^{2}>0.8\right)$ of GWASidentified TASs. We identified $15.7 \%$ of 344 emsQTLs (SNPs only and not indels) to be strongly associated with 145 GWAS hits (Supplemental Table S5), an significant enrichment of emsQTLs in GWAS TASs comparing with background SNPs for both the miRNA seed binding site $(P=7.54 \mathrm{E}-28$, hypergeometric test $)$ and differentiated miRNA binding signals $(P=3.30 \mathrm{E}-4$, hypergeometric test) (Supplemental Table S6). Most of these 145 GWAS index SNPs locate in introns or intergenic regions with poorly annotated functions (Supplemental Table S7). Therefore, our emsQTL analysis could potentially identify causal mechanisms underlying disease/trait SNPs. Interestingly, phenotypes associated with these 145 GWAS hits are mostly related to autoimmune diseases and blood cell traits (Supplemental Table S7), suggesting the effect of emsQTLs to be driven by cell type specificity of the lymphoblastoid cells in the Geuvadis data.

\section{Functional Effect of emsQTLs in ceRNA Regulatory Network}

\section{Prioritization of emsQTLs}

To comprehensively evaluate the association between emsQTLs and ceRNA regulation, we rely not only on the statistical significance, but also on the magnitude of emsQTLs function on titrating miRNA availability and ceRNA-dependent gene expression changes. Several factors have been reported to influence ceRNA effectiveness, including miRNA and ceRNA expression level, the binding affinity of MRE, as well as the positive correlation between ceRNAs 
expression $^{3 ; 5}$ 59. We attempted to prioritize the functional emsQTLs according to these factors. Since our regression model has already accounted for confounding factors from miRNA expression variation in the emsQTL calling step, we therefore only focused on ceRNA-related factors in the prioritization. We first calculated the degree of gene expression change on ceD and ceT in different genotypes, which can be measured by the sum of $\log \left|\beta_{d}\right|$ and $\log \left|\beta_{t}\right|$. We further required consistent direction between $\beta_{d}$ and the $\Delta$ context+ functional prediction score from TargetScan. Finally, we asked for positive correlation (>0.1) for ceD and ceT in the specific homozygous emsQTL genotypes, when ceD and ceT actively compete for miRNA binding. Based on aforementioned criteria, we successfully identified 239 variant-miRNA-ceDceT units for 93 unique emsQTLs with sufficient functional evidences (Supplemental Table S8). The top variant rs3208409 creates a miR-940-3p binding site in the 3'UTR of HLA-DRB1 gene, which competes with L3MBTL2 for miR-940-3p binding ( $P=7.94 \mathrm{E}-27)$. The large effect of rs3208409 on the gene expression of $\operatorname{ceD}\left(\beta_{d}=-115.18\right)$ and ceT $\left(\beta_{t}=1.14\right)$, the consistent functional prediction $(\Delta$ context+ score $=-0.21)$, and the high correlation $($ Cor $=0.38)$ between ceD and ceT in homozygote individuals provide robust evidences of the causality of this emsQTL (Supplemental Figure S10). We further overlapped this prioritized list with GWAS signals and identified 21 phenotype-associated variant-miRNA-ceD-ceT units (Supplemental Table S9).

\section{Genetic effect on ceRNA regulatory network}

The altered expression of individual genes might affect the expression of many other genes in the whole ceRNA regulatory network by the miRNA sponge mechanism ${ }^{60 ; 61}$. From the 1,875 significant variant-miRNA-ceD-ceT units our model identified, we constructed the global ceRNA regulatory network under the control of 387 independent emsQTLs in the EUR population 
(Supplemental Figure S11). We also generated the network for 21 phenotype-associated variant-miRNA-ceD-ceT units (Supplemental Figure S12). Majority of ceDs can be associated with more than one ceTs by single genetic effect. For example, rs11540855 on ceD ABHD8 could influence the expression of two ceTs AXIN1 and RPRM through competing for binding to miR-4707-3p (Figure 6A), and the expression of $A B H D 8$ and its two ceTs are positively correlated under the active genotype GG (Figure 6B-D). Interestingly, emsQTL rs11540855 located in the 3' UTR of $A B H D 8$ on 19p13 has been recently reported to have top significant association with breast cancer risk (GWAS $P=1.65 \mathrm{E}-09$ ) after genotype imputation ${ }^{62 ; 63}$. In addition, AXIN1 and RPRM were recently reported as tumor suppressors in breast cancer development ${ }^{64}$, and miR-4707-3p is highly expressed in breast cancer ${ }^{65}$. These evidences suggest that emsQTL rs11540855 might influence breast cancer developments by regulating tumor suppressors $A X I N 1$ and RPRM through the ceRNA pathway. In addition to the aforementioned regulatory relationship, one ceD can also be regulated by multiple miRNAs, and a single miRNA can regulate multiple ceDs and ceTs through different emsQTLs. These interactions highlight the complexity of genetic effect on ceRNA regulatory network.

\section{Discussion}

In this study, we, for the first time, integrated the 1000 Genomes genotype and Geuvadis RNA sequencing data to investigate the effect of human genetic variations on ceRNA regulation. Using a multivariate linear model, we successfully identified hundreds of emsQTLs and related ceDs/ceTs at the genome-wide level. We found that recent natural selection is shaping many emsQTLs in different human populations. Functional analysis of these genetic variants indicated that most of emsQTLs are functionally relevant to important biological processes and are significantly enriched in GWAS risk loci. Furthermore, we prioritized these loci with their associated ceRNAs according to different criteria and evaluated their collective effect on the 
ceRNA regulatory network. Our study provides a novel angle to interpret genetic effect in posttranscriptional gene regulation.

Although our regression model already considered many candidate confounding factors, such as miRNA expression level, ceRNA expression variability, as well as population genetic structure, there may still be missing factors that impact the performance and statistical power in the emsQTL detection. One potential limitation is that we only treated each pair of ceD and ceT as an independent test unit in the local miRNA-centered regulatory network instead of modeling the whole ceRNA regulatory network. Recent studies have shown that a small perturbation of ceRNA expression usually shifts the equilibrium of ceRNA regulatory network especially when concentrations of miRNAs and targets are comparable ${ }^{66}$. The cascade effect from miRNA redistribution and ceRNA competition in the global level $8 ; 67$ requires a complete and complex mathematical model to accurately describe full responses of the whole network. Another limitation of our study is that current computational predictions of miRNA binding sites still have inadequate performances ${ }^{68}$. To balance sensitivity and specificity of miRNA target prediction, we chose to use a strict context+ score threshold from TargetScan predictions, instead of the union or intersection of multiple miRNA-target prediction algorithms such as TargetScan, PITA $^{36}$, miRanda ${ }^{69}$, etc. Therefore, it is likely that our emsQTLs detection missed some causal variants not predicted by TargetScan. Future experiments such as CLIP-seq, if done on individuals, could better capture miRNA-target interactions and improve our emsQTLs inference.

Using genetic and transcriptomic data from different populations, we found many populationspecific emsQTLs and identified putative loci undergoing recent positive selection. These results represent a useful supplement to studies of recent natural selection of human miRNA targets ${ }^{70-}$ 72 and significantly extent functional categories for positively selected loci ${ }^{73}$. Currently, available transcriptome profiles of five subpopulation from Geuvadis, of which majority are from European populations, prevent the inference of positive selection signals in other human races such as 
Asian and Native American populations. The recent human Genotype-Tissue Expression (GTEx) project has produced large-scale transcriptome profiles in multiple tissues of hundreds of donors $^{74}$, which provides new opportunities to study tissue-specific associations between genetic variations and ceRNA regulation.

Since many genes contain multiple binding sites of the same miRNA, some might suspect single MRE perturbations to have small effects on ceRNA expression and the downstream miRNA regulatory network. This is not surprising, considering that most QTLs usually also only account for a small fraction of the total genetic heritability in the population. However, although many QTLs individually exert relatively small effects, together they might contribute to a significant complex trait $^{75}$. Theoretical simulations and quantitative experiments have demonstrated that some perturbations on individual miRNA binding site can indeed affect the entire ceRNA regulatory network ${ }^{8 ; 76 ; 77}$. Our emsQTL analyses on human populations suggest that DNA polymorphisms affecting ceRNA regulation is a widespread phenomenon in the human evolution and contribute significantly to complex traits.

\section{Description of Supplemental Data}

Supplemental Data include twelve figures and nine tables.

\section{Acknowledgement}

We are very grateful to Dr. Graham McVicker for his discussions and suggestions. The project was supported by funds from Y S and Christabel Lung Postgraduate Scholarship (MJL), Research Grants Council, Hong Kong SAR, China 17121414M (JWW), The National Institute of Health U41 HG7000 (XSL) and The National Institute of Health R01 GM113242-01 (JSL). 
bioRxiv preprint doi: https://doi.org/10.1101/016865; this version posted March 22, 2015. The copyright holder for this preprint (which was not certified by peer review) is the author/funder, who has granted bioRxiv a license to display the preprint in perpetuity. It is made available under aCC-BY-NC-ND 4.0 International license. 


\section{Reference}

1. Tay, Y., Rinn, J., and Pandolfi, P.P. (2014). The multilayered complexity of ceRNA crosstalk and competition. Nature 505, 344-352.

2. Kartha, R.V., and Subramanian, S. (2014). Competing endogenous RNAs (ceRNAs): new entrants to the intricacies of gene regulation. Frontiers in genetics 5,8 .

3. Ebert, M.S., and Sharp, P.A. (2010). Emerging roles for natural microRNA sponges. Current biology : CB 20, R858-861.

4. Karreth, F.A., and Pandolfi, P.P. (2013). ceRNA cross-talk in cancer: when ce-bling rivalries go awry. Cancer discovery 3, 1113-1121.

5. Salmena, L., Poliseno, L., Tay, Y., Kats, L., and Pandolfi, P.P. (2011). A ceRNA hypothesis: the Rosetta Stone of a hidden RNA language? Cell 146, 353-358.

6. Kumar, M.S., Armenteros-Monterroso, E., East, P., Chakravorty, P., Matthews, N., Winslow, M.M., and Downward, J. (2014). HMGA2 functions as a competing endogenous RNA to promote lung cancer progression. Nature 505, 212-217.

7. Li, L., Wang, D., Xue, M., Mi, X., Liang, Y., and Wang, P. (2014). 3'UTR shortening identifies high-risk cancers with targeted dysregulation of the ceRNA network. Scientific reports 4, 5406.

8. Ala, U., Karreth, F.A., Bosia, C., Pagnani, A., Taulli, R., Leopold, V., Tay, Y., Provero, P., Zecchina, R., and Pandolfi, P.P. (2013). Integrated transcriptional and competitive endogenous RNA networks are cross-regulated in permissive molecular environments. Proceedings of the National Academy of Sciences of the United States of America 110, 7154-7159.

9. Hanin, G., Shenhar-Tsarfaty, S., Yayon, N., Hoe, Y.Y., Bennett, E.R., Sklan, E.H., Rao, D.C., Rankinen, T., Bouchard, C., Geifman-Shochat, S., et al. (2014). Competing targets of microRNA-608 affect anxiety and hypertension. Human molecular genetics $23,4569-4580$. 
10. Cheung, V.G., Conlin, L.K., Weber, T.M., Arcaro, M., Jen, K.Y., Morley, M., and Spielman, R.S. (2003).

Natural variation in human gene expression assessed in lymphoblastoid cells. Nature genetics 33, $422-425$.

11. McDaniell, R., Lee, B.K., Song, L., Liu, Z., Boyle, A.P., Erdos, M.R., Scott, L.J., Morken, M.A., Kucera, K.S., Battenhouse, A., et al. (2010). Heritable individual-specific and allele-specific chromatin signatures in humans. Science 328, 235-239.

12. Li, M.J., Yan, B., Sham, P.C., and Wang, J. (2014). Exploring the function of genetic variants in the non-coding genomic regions: approaches for identifying human regulatory variants affecting gene expression. Briefings in bioinformatics.

13. Gamazon, E.R., Innocenti, F., Wei, R., Wang, L., Zhang, M., Mirkov, S., Ramirez, J., Huang, R.S., Cox, N.J., Ratain, M.J., et al. (2013). A genome-wide integrative study of microRNAs in human liver. BMC genomics 14, 395.

14. Civelek, M., Hagopian, R., Pan, C., Che, N., Yang, W.P., Kayne, P.S., Saleem, N.K., Cederberg, H., Kuusisto, J., Gargalovic, P.S., et al. (2013). Genetic regulation of human adipose microRNA expression and its consequences for metabolic traits. Human molecular genetics 22, 3023-3037.

15. Siddle, K.J., Deschamps, M., Tailleux, L., Nedelec, Y., Pothlichet, J., Lugo-Villarino, G., Libri, V., Gicquel, B., Neyrolles, O., Laval, G., et al. (2014). A genomic portrait of the genetic architecture and regulatory impact of microRNA expression in response to infection. Genome research 24, 850859.

16. Lappalainen, T., Sammeth, M., Friedlander, M.R., t Hoen, P.A., Monlong, J., Rivas, M.A., GonzalezPorta, M., Kurbatova, N., Griebel, T., Ferreira, P.G., et al. (2013). Transcriptome and genome sequencing uncovers functional variation in humans. Nature $501,506-511$. 
17. Gamazon, E.R., Ziliak, D., Im, H.K., LaCroix, B., Park, D.S., Cox, N.J., and Huang, R.S. (2012). Genetic architecture of microRNA expression: implications for the transcriptome and complex traits. American journal of human genetics 90, 1046-1063.

18. Lu, J., and Clark, A.G. (2012). Impact of microRNA regulation on variation in human gene expression. Genome research 22, 1243-1254.

19. Genomes Project, C., Abecasis, G.R., Auton, A., Brooks, L.D., DePristo, M.A., Durbin, R.M., Handsaker, R.E., Kang, H.M., Marth, G.T., and McVean, G.A. (2012). An integrated map of genetic variation from 1,092 human genomes. Nature 491, 56-65.

20. Harrow, J., Frankish, A., Gonzalez, J.M., Tapanari, E., Diekhans, M., Kokocinski, F., Aken, B.L., Barrell, D., Zadissa, A., Searle, S., et al. (2012). GENCODE: the reference human genome annotation for The ENCODE Project. Genome research 22, 1760-1774.

21. Kozomara, A., and Griffiths-Jones, S. (2014). miRBase: annotating high confidence microRNAs using deep sequencing data. Nucleic acids research 42, D68-73.

22. Lewis, B.P., Burge, C.B., and Bartel, D.P. (2005). Conserved seed pairing, often flanked by adenosines, indicates that thousands of human genes are microRNA targets. Cell 120, 15-20.

23. Lorenz, R., Bernhart, S.H., Honer Zu Siederdissen, C., Tafer, H., Flamm, C., Stadler, P.F., and Hofacker, I.L. (2011). ViennaRNA Package 2.0. Algorithms for molecular biology : AMB 6, 26.

24. Stegle, O., Parts, L., Piipari, M., Winn, J., and Durbin, R. (2012). Using probabilistic estimation of expression residuals (PEER) to obtain increased power and interpretability of gene expression analyses. Nature protocols 7, 500-507.

25. Huang da, W., Sherman, B.T., and Lempicki, R.A. (2009). Systematic and integrative analysis of large gene lists using DAVID bioinformatics resources. Nature protocols 4, 44-57.

26. Bruno, A.E., Li, L., Kalabus, J.L., Pan, Y., Yu, A., and Hu, Z. (2012). miRdSNP: a database of diseaseassociated SNPs and microRNA target sites on 3'UTRs of human genes. BMC genomics 13, 44. 
27. Li, M.J., and Wang, J. (2014). Current trend of annotating single nucleotide variation in humans - a case study on SNVrap. Methods.

28. Li, M.J., Wang, P., Liu, X., Lim, E.L., Wang, Z., Yeager, M., Wong, M.P., Sham, P.C., Chanock, S.J., and Wang, J. (2012). GWASdb: a database for human genetic variants identified by genome-wide association studies. Nucleic acids research 40, D1047-1054.

29. Grossman, S.R., Shlyakhter, I., Karlsson, E.K., Byrne, E.H., Morales, S., Frieden, G., Hostetter, E., Angelino, E., Garber, M., Zuk, O., et al. (2010). A composite of multiple signals distinguishes causal variants in regions of positive selection. Science $327,883-886$.

30. Weir, B.S., and Hill, W.G. (2002). Estimating F-statistics. Annual review of genetics 36, 721-750.

31. Tajima, F. (1989). Statistical method for testing the neutral mutation hypothesis by DNA polymorphism. Genetics 123, 585-595.

32. Voight, B.F., Kudaravalli, S., Wen, X., and Pritchard, J.K. (2006). A map of recent positive selection in the human genome. PLoS biology 4, e72.

33. Sabeti, P.C., Varilly, P., Fry, B., Lohmueller, J., Hostetter, E., Cotsapas, C., Xie, X., Byrne, E.H., McCarroll, S.A., Gaudet, R., et al. (2007). Genome-wide detection and characterization of positive selection in human populations. Nature 449, 913-918.

34. Chen, H., Patterson, N., and Reich, D. (2010). Population differentiation as a test for selective sweeps. Genome research 20, 393-402.

35. Leslie, R., O'Donnell, C.J., and Johnson, A.D. (2014). GRASP: analysis of genotype-phenotype results from 1390 genome-wide association studies and corresponding open access database. Bioinformatics 30, i185-194.

36. Kertesz, M., lovino, N., Unnerstall, U., Gaul, U., and Segal, E. (2007). The role of site accessibility in microRNA target recognition. Nature genetics 39, 1278-1284. 
37. Yu, W., Gwinn, M., Clyne, M., Yesupriya, A., and Khoury, M.J. (2008). A navigator for human genome epidemiology. Nature genetics 40, 124-125.

38. Ramos, E.M., Hoffman, D., Junkins, H.A., Maglott, D., Phan, L., Sherry, S.T., Feolo, M., and Hindorff, L.A. (2014). Phenotype-Genotype Integrator (PheGenl): synthesizing genome-wide association study (GWAS) data with existing genomic resources. European journal of human genetics : EJHG 22, 144-147.

39. Chen, M., Masaki, T., and Sawamura, T. (2002). LOX-1, the receptor for oxidized low-density lipoprotein identified from endothelial cells: implications in endothelial dysfunction and atherosclerosis. Pharmacology \& therapeutics 95, 89-100.

40. Lambert, J.C., Luedecking-Zimmer, E., Merrot, S., Hayes, A., Thaker, U., Desai, P., Houzet, A., Hermant, X., Cottel, D., Pritchard, A., et al. (2003). Association of 3'-UTR polymorphisms of the oxidised LDL receptor 1 (OLR1) gene with Alzheimer's disease. Journal of medical genetics 40, 424-430.

41. Khaidakov, M., Mitra, S., Kang, B.Y., Wang, X., Kadlubar, S., Novelli, G., Raj, V., Winters, M., Carter, W.C., and Mehta, J.L. (2011). Oxidized LDL receptor 1 (OLR1) as a possible link between obesity, dyslipidemia and cancer. PloS one 6, e20277.

42. Bhattacharya, A., Ziebarth, J.D., and Cui, Y. (2014). PolymiRTS Database 3.0: linking polymorphisms in microRNAs and their target sites with human diseases and biological pathways. Nucleic acids research 42, D86-91.

43. Gong, J., Tong, Y., Zhang, H.M., Wang, K., Hu, T., Shan, G., Sun, J., and Guo, A.Y. (2012). Genomewide identification of SNPs in microRNA genes and the SNP effects on microRNA target binding and biogenesis. Human mutation 33, 254-263.

44. Wang, S., and Olson, E.N. (2009). AngiomiRs--key regulators of angiogenesis. Current opinion in genetics \& development 19, 205-211. 
45. Anand, S., and Cheresh, D.A. (2011). MicroRNA-mediated regulation of the angiogenic switch.

Current opinion in hematology 18, 171-176.

46. Bonauer, A., Boon, R.A., and Dimmeler, S. (2010). Vascular microRNAs. Current drug targets 11, 943949.

47. Wurdinger, T., Tannous, B.A., Saydam, O., Skog, J., Grau, S., Soutschek, J., Weissleder, R., Breakefield, X.O., and Krichevsky, A.M. (2008). miR-296 regulates growth factor receptor overexpression in angiogenic endothelial cells. Cancer cell 14, 382-393.

48. Yoon, A.R., Gao, R., Kaul, Z., Choi, I.K., Ryu, J., Noble, J.R., Kato, Y., Saito, S., Hirano, T., Ishii, T., et al. (2011). MicroRNA-296 is enriched in cancer cells and downregulates p21WAF1 mRNA expression via interaction with its 3' untranslated region. Nucleic acids research 39, 8078-8091.

49. Garcia-Domingo, D., Leonardo, E., Grandien, A., Martinez, P., Albar, J.P., Izpisua-Belmonte, J.C., and Martinez, A.C. (1999). DIO-1 is a gene involved in onset of apoptosis in vitro, whose misexpression disrupts limb development. Proceedings of the National Academy of Sciences of the United States of America 96, 7992-7997.

50. Stark, A.L., Hause, R.J., Jr., Gorsic, L.K., Antao, N.N., Wong, S.S., Chung, S.H., Gill, D.F., Im, H.K., Myers, J.L., White, K.P., et al. (2014). Protein quantitative trait loci identify novel candidates modulating cellular response to chemotherapy. PLoS genetics 10, e1004192.

51. Futterer, A., Campanero, M.R., Leonardo, E., Criado, L.M., Flores, J.M., Hernandez, J.M., San Miguel, J.F., and Martinez, A.C. (2005). Dido gene expression alterations are implicated in the induction of hematological myeloid neoplasms. The Journal of clinical investigation 115, 2351-2362.

52. Shi, T.Y., Cheng, X., Yu, K.D., Sun, M.H., Shao, Z.M., Wang, M.Y., Zhu, M.L., He, J., Li, Q.X., Chen, X.J., et al. (2013). Functional variants in TNFAIP8 associated with cervical cancer susceptibility and clinical outcomes. Carcinogenesis 34, 770-778. 
53. Kumar, D., Whiteside, T.L., and Kasid, U. (2000). Identification of a novel tumor necrosis factor-alphainducible gene, SCC-S2, containing the consensus sequence of a death effector domain of fasassociated death domain-like interleukin- 1beta-converting enzyme-inhibitory protein. The Journal of biological chemistry 275, 2973-2978.

54. Liu, T., Gao, H., Chen, X., Lou, G., Gu, L., Yang, M., Xia, B., and Yin, H. (2013). TNFAIP8 as a predictor of metastasis and a novel prognostic biomarker in patients with epithelial ovarian cancer. British journal of cancer 109, 1685-1692.

55. Scott, S.A., Edelmann, L., Kornreich, R., and Desnick, R.J. (2008). Warfarin pharmacogenetics: CYP2C9 and VKORC1 genotypes predict different sensitivity and resistance frequencies in the Ashkenazi and Sephardi Jewish populations. American journal of human genetics 82, 495-500.

56. Kringen, M.K., Haug, K.B., Grimholt, R.M., Stormo, C., Narum, S., Opdal, M.S., Fosen, J.T., Piehler, A.P., Johansen, P.W., Seljeflot, I., et al. (2011). Genetic variation of VKORC1 and CYP4F2 genes related to warfarin maintenance dose in patients with myocardial infarction. Journal of biomedicine \& biotechnology 2011, 739751.

57. Limdi, N.A., Beasley, T.M., Crowley, M.R., Goldstein, J.A., Rieder, M.J., Flockhart, D.A., Arnett, D.K., Acton, R.T., and Liu, N. (2008). VKORC1 polymorphisms, haplotypes and haplotype groups on warfarin dose among African-Americans and European-Americans. Pharmacogenomics 9, 14451458.

58. Rieder, M.J., Reiner, A.P., Gage, B.F., Nickerson, D.A., Eby, C.S., McLeod, H.L., Blough, D.K., Thummel, K.E., Veenstra, D.L., and Rettie, A.E. (2005). Effect of VKORC1 haplotypes on transcriptional regulation and warfarin dose. The New England journal of medicine 352, 2285-2293.

59. Mukherji, S., Ebert, M.S., Zheng, G.X., Tsang, J.S., Sharp, P.A., and van Oudenaarden, A. (2011). MicroRNAs can generate thresholds in target gene expression. Nature genetics 43, 854-859. 
60. Sumazin, P., Yang, X., Chiu, H.S., Chung, W.J., Iyer, A., Llobet-Navas, D., Rajbhandari, P., Bansal, M., Guarnieri, P., Silva, J., et al. (2011). An extensive microRNA-mediated network of RNA-RNA interactions regulates established oncogenic pathways in glioblastoma. Cell 147, 370-381.

61. Bosia, C., Pagnani, A., and Zecchina, R. (2013). Modelling Competing Endogenous RNA Networks. PloS one 8, e66609.

62. Antoniou, A.C., Wang, X., Fredericksen, Z.S., McGuffog, L., Tarrell, R., Sinilnikova, O.M., Healey, S., Morrison, J., Kartsonaki, C., Lesnick, T., et al. (2010). A locus on 19p13 modifies risk of breast cancer in BRCA1 mutation carriers and is associated with hormone receptor-negative breast cancer in the general population. Nature genetics $42,885-892$.

63. Stevens, K.N., Fredericksen, Z., Vachon, C.M., Wang, X., Margolin, S., Lindblom, A., Nevanlinna, H., Greco, D., Aittomaki, K., Blomqvist, C., et al. (2012). 19p13.1 is a triple-negative-specific breast cancer susceptibility locus. Cancer research 72, 1795-1803.

64. Zhang, X., Farrell, A.S., Daniel, C.J., Arnold, H., Scanlan, C., Laraway, B.J., Janghorban, M., Lum, L., Chen, D., Troxell, M., et al. (2012). Mechanistic insight into Myc stabilization in breast cancer involving aberrant Axin1 expression. Proceedings of the National Academy of Sciences of the United States of America 109, 2790-2795.

65. Persson, H., Kvist, A., Rego, N., Staaf, J., Vallon-Christersson, J., Luts, L., Loman, N., Jonsson, G., Naya, H., Hoglund, M., et al. (2011). Identification of new microRNAs in paired normal and tumor breast tissue suggests a dual role for the ERBB2/Her2 gene. Cancer research 71, 78-86.

66. Levine, E., Zhang, Z., Kuhlman, T., and Hwa, T. (2007). Quantitative characteristics of gene regulation by small RNA. PLoS biology 5, e229.

67. Seitz, H. (2009). Redefining microRNA targets. Current biology : CB 19, 870-873.

68. Pasquinelli, A.E. (2012). MicroRNAs and their targets: recognition, regulation and an emerging reciprocal relationship. Nature reviews Genetics 13, 271-282. 
69. John, B., Enright, A.J., Aravin, A., Tuschl, T., Sander, C., and Marks, D.S. (2004). Human MicroRNA targets. PLoS biology 2, e363.

70. Chen, K., and Rajewsky, N. (2006). Natural selection on human microRNA binding sites inferred from SNP data. Nature genetics 38, 1452-1456.

71. Li, J., Liu, Y., Xin, X., Kim, T.S., Cabeza, E.A., Ren, J., Nielsen, R., Wrana, J.L., and Zhang, Z. (2012).

Evidence for positive selection on a number of MicroRNA regulatory interactions during recent human evolution. PLoS genetics 8, e1002578.

72. Saunders, M.A., Liang, H., and Li, W.H. (2007). Human polymorphism at microRNAs and microRNA target sites. Proceedings of the National Academy of Sciences of the United States of America 104, 3300-3305.

73. Grossman, S.R., Andersen, K.G., Shlyakhter, I., Tabrizi, S., Winnicki, S., Yen, A., Park, D.J., Griesemer, D., Karlsson, E.K., Wong, S.H., et al. (2013). Identifying recent adaptations in large-scale genomic data. Cell 152, 703-713.

74. Consortium, G.T. (2013). The Genotype-Tissue Expression (GTEx) project. Nature genetics 45, 580585.

75. Mackay, T.F., Stone, E.A., and Ayroles, J.F. (2009). The genetics of quantitative traits: challenges and prospects. Nature reviews Genetics 10, 565-577.

76. Xie, P., Liu, Y., Li, Y., Zhang, M.Q., and Wang, X. (2014). MIROR: a method for cell-type specific microRNA occupancy rate prediction. Molecular bioSystems 10, 1377-1384.

77. Bosson, Andrew D., Zamudio, Jesse R., and Sharp, Phillip A. Endogenous miRNA and Target Concentrations Determine Susceptibility to Potential ceRNA Competition. Molecular cell 56, 347-359. 
78. Teichert, M., Eijgelsheim, M., Rivadeneira, F., Uitterlinden, A.G., van Schaik, R.H., Hofman, A., De Smet, P.A., van Gelder, T., Visser, L.E., and Stricker, B.H. (2009). A genome-wide association study of acenocoumarol maintenance dosage. Human molecular genetics 18, 3758-3768.

79. Marzi, C., Albrecht, E., Hysi, P.G., Lagou, V., Waldenberger, M., Tonjes, A., Prokopenko, I., Heim, K., Blackburn, H., Ried, J.S., et al. (2010). Genome-wide association study identifies two novel regions at 11p15.5-p13 and 1p31 with major impact on acute-phase serum amyloid A. PLoS genetics 6, e1001213.

80. Naccarati, A., Pardini, B., Stefano, L., Landi, D., Slyskova, J., Novotny, J., Levy, M., Polakova, V., Lipska, L., and Vodicka, P. (2012). Polymorphisms in miRNA-binding sites of nucleotide excision repair genes and colorectal cancer risk. Carcinogenesis 33, 1346-1351.

81. Kettunen, J., Tukiainen, T., Sarin, A.P., Ortega-Alonso, A., Tikkanen, E., Lyytikainen, L.P., Kangas, A.J., Soininen, P., Wurtz, P., Silander, K., et al. (2012). Genome-wide association study identifies multiple loci influencing human serum metabolite levels. Nature genetics 44, 269-276.

82. Cusanovich, D.A., Billstrand, C., Zhou, X., Chavarria, C., De Leon, S., Michelini, K., Pai, A.A., Ober, C., and Gilad, Y. (2012). The combination of a genome-wide association study of lymphocyte count and analysis of gene expression data reveals novel asthma candidate genes. Human molecular genetics 21, 2111-2123.

83. Inouye, M., Ripatti, S., Kettunen, J., Lyytikainen, L.P., Oksala, N., Laurila, P.P., Kangas, A.J., Soininen, P., Savolainen, M.J., Viikari, J., et al. (2012). Novel Loci for metabolic networks and multi-tissue expression studies reveal genes for atherosclerosis. PLoS genetics 8, e1002907.

84. Hong, M.G., Karlsson, R., Magnusson, P.K., Lewis, M.R., Isaacs, W., Zheng, L.S., Xu, J., Gronberg, H., Ingelsson, E., Pawitan, Y., et al. (2013). A genome-wide assessment of variability in human serum metabolism. Human mutation 34, 515-524. 
85. Hwang, S.J., Yang, Q., Meigs, J.B., Pearce, E.N., and Fox, C.S. (2007). A genome-wide association for kidney function and endocrine-related traits in the NHLBI's Framingham Heart Study. BMC medical genetics 8 Suppl 1, S10.

86. Pare, G., Chasman, D.I., Kellogg, M., Zee, R.Y., Rifai, N., Badola, S., Miletich, J.P., and Ridker, P.M. (2008). Novel association of ABO histo-blood group antigen with soluble ICAM-1: results of a genome-wide association study of 6,578 women. PLoS genetics 4, e1000118.

87. Rizk, N.M., and Derbala, M.F. (2013). Genetic polymorphisms of ICAM 1 and IL28 as predictors of liver fibrosis severity and viral clearance in hepatitis $C$ genotype 4 . Clinics and research in hepatology and gastroenterology 37, 262-268.

88. Waterworth, D.M., Ricketts, S.L., Song, K., Chen, L., Zhao, J.H., Ripatti, S., Aulchenko, Y.S., Zhang, W., Yuan, X., Lim, N., et al. (2010). Genetic variants influencing circulating lipid levels and risk of coronary artery disease. Arteriosclerosis, thrombosis, and vascular biology 30, 2264-2276.

89. van der Harst, P., Zhang, W., Mateo Leach, I., Rendon, A., Verweij, N., Sehmi, J., Paul, D.S., Elling, U., Allayee, H., Li, X., et al. (2012). Seventy-five genetic loci influencing the human red blood cell. Nature 492, 369-375.

90. Guevara-Cruz, M., Lai, C.Q., Richardson, K., Parnell, L.D., Lee, Y.C., Tovar, A.R., Ordovas, J.M., and Torres, N. (2013). Effect of a GFOD2 variant on responses in total and LDL cholesterol in Mexican subjects with hypercholesterolemia after soy protein and soluble fiber supplementation. Gene $532,211-215$.

91. Wu, C., Kraft, P., Zhai, K., Chang, J., Wang, Z., Li, Y., Hu, Z., He, Z., Jia, W., Abnet, C.C., et al. (2012). Genome-wide association analyses of esophageal squamous cell carcinoma in Chinese identify multiple susceptibility loci and gene-environment interactions. Nature genetics 44, 1090-1097.

92. Hinds, D.A., McMahon, G., Kiefer, A.K., Do, C.B., Eriksson, N., Evans, D.M., St Pourcain, B., Ring, S.M., Mountain, J.L., Francke, U., et al. (2013). A genome-wide association meta-analysis of self- 
reported allergy identifies shared and allergy-specific susceptibility loci. Nature genetics $45,907-$

911.

93. Farh, K.K., Marson, A., Zhu, J., Kleinewietfeld, M., Housley, W.J., Beik, S., Shoresh, N., Whitton, H., Ryan, R.J., Shishkin, A.A., et al. (2014). Genetic and epigenetic fine mapping of causal autoimmune disease variants. Nature.

94. McGue, M., Zhang, Y., Miller, M.B., Basu, S., Vrieze, S., Hicks, B., Malone, S., Oetting, W.S., and lacono, W.G. (2013). A genome-wide association study of behavioral disinhibition. Behavior genetics 43, 363-373. 


\section{Figure legends}

Figure 1: Circos plot of all detected emsQTLs. Features or glyphs are displayed from the outer to the inner, include the number of chromosome, the chromosome ideograms, copy number variation hotspots (red region), Manhattan plot for emsQTLs with -log10( $P$-value), Manhattan plot for GWAS TASs in miRNA binding site predicted by TargetScan, genome variant density (red: dbSNP, black: 1000 Genomes, purple: HapMap 3), OMIM gene distribution and diseasesusceptible region distribution.

Figure 2: Genome-wide detection of emsQTLs. (A) The proportion of emsQTLs for SNP and indel. (B) The overlapping of emsQTLs in Geuvadis eQTLs. (C) Venn diagram of emsQTLs in European populations. (D) Venn diagram of emsQTL between European and African populations.

Figure 3: The positive selection of emsQTLs. (A) The hierarchical clustering of according to derived allele frequency for 46 putatively positive selected emsQTLs in different population. (B) iHS scores in rs1050286 locus for YRI. (C) XPEHH scores in rs1050286 locus for YRI.

Figure 4: The functional properties of emsQTLs for EUR population. (A) The distribution of $\beta_{d}$. (B) The distribution of $\beta_{t}$. (C) The direction concordance between association and functional prediction for all emsQTLs.

Figure 5: The genetic effect of rs1056984 in ceRNA regulation for EUR population. (A) Hybridization pattern between miR-296-5p and binding site of DIDO1 on derived allele A. (B) Hybridization pattern between miR-296-5p and binding site of DIDO1 on ancestral allele G. (C) Boxplot of gene expression of DIDO1 on different genotype. (D) Boxplot of gene expression of TNFAIP8L1 on different genotypes. (E) The gene expression correlation of DIDO1 and TNFAIP8L1 under different genotypes. (F) Schematic diagram for rs1056984 affecting ceRNA competition under different alleles, it impacts the expression of tumor suppressor and oncogene in a reciprocal and coordinate manner. MFE: minimum free energy.

Figure 6: The genetic effect of $r s 11540855$ in ceRNA regulation for EUR population. (A) Small ceRNA regulatory network driven by rs 11540855 , red circle: ceDs; yellow triangle: miRNA; bule circle: ceTs; red suppression line: the miRNA-ceD regulation, $G$ for gain-of-function mutation; gray suppression line: the miRNA-ceT regulation; orange arrow: ceD activate ceTs in gain-offunction situation $\left(\beta_{\mathrm{d}}<0\right.$ and $\beta_{\mathrm{t}}>0$ ). (B) Boxplot of gene expression of $A B H D 8$ on different genotypes. (C) Boxplot of gene expression of $A X I N 1$ on different genotypes and the correlation with $A B H D 8$ on genotype GG. (C) Boxplot of gene expression of RPRM on different genotypes and the correlation with $A B H D 8$ on genotype GG. 


\section{Tables}

Table 1: The emsQTLs that overlap with GWAS leading TASs

\begin{tabular}{|c|c|c|c|c|c|c|c|c|c|c|c|}
\hline Chr & Pos & SNP & Ref & Alt & $\begin{array}{l}\text { emsQTL } \\
P \text {-value }\end{array}$ & ceD & ceTs & miRNA & Effect $^{b}$ & $\begin{array}{c}\text { GWAS } \\
P \text {-value }\end{array}$ & GWAS Traits \\
\hline 16 & 31102321 & rs7294 & C & $\mathrm{T}$ & $1.37 \mathrm{E}-06$ & VKORC1 & $\begin{array}{c}\text { EIF2B5, RPTOR, } \\
\text { LRRFIP1 }\end{array}$ & miR-147a & Gain & $1.40 \mathrm{E}-45$ & $\begin{array}{l}\text { Warfarin and acenocoumarol } \\
\text { maintenance dosage } \mathrm{e}^{58 ; 78}\end{array}$ \\
\hline 11 & 18388128 & rs4596 & G & C & $1.15 \mathrm{E}-05$ & GTF2H1 & $\begin{array}{c}\text { TSTD2, TMOD1, } \\
\text { TOB1, PPARGC1A, } \\
\text { KLF5 }\end{array}$ & miR-642a-5p & Loss & $2.17 \mathrm{E}-35$ & $\begin{array}{l}\text { Amyloid A Levels }{ }^{79}, \text { Colorectal } \\
\text { cancer }\end{array}$ \\
\hline 12 & 56863770 & rs2657880 & G & C & $1.87 \mathrm{E}-51$ & SPRYD4 & XKR6, AC004985.2 & miR-3157-5p & Loss & $2.28 \mathrm{E}-31$ & $\begin{array}{l}\text { Serum metabolite levels }{ }^{81} \\
\text { Lymphocyte counts }^{82} \\
\text { Metabolite levels }\end{array}$ \\
\hline 10 & 100176869 & rs701801 & C & $\mathrm{T}$ & $4.70 \mathrm{E}-13$ & HPS1 & TRIOBP, KLHL3O & miR-491-5p & Loss & $1.34 \mathrm{E}-25$ & $\begin{array}{c}\text { Serum metabolism }{ }^{84} \text {, Endocrine } \\
\text { traits }^{85}\end{array}$ \\
\hline 19 & 10397238 & rs281437 & C & $\mathrm{T}$ & 0.00019 & ICAM1 & $\begin{array}{l}\text { C16orf54, } \\
\text { FBXO41, SH2D4A }\end{array}$ & miR-3667-5p & Loss & $3.00 \mathrm{E}-10$ & $\begin{array}{c}\text { Soluble intercellular adhesion } \\
\text { molecule } 1 \text { level }^{86} \text {, hepatic } \\
\text { fibrosis }\end{array}$ \\
\hline 16 & 67708897 & rs12449157 & A & G & 7.69E-06 & GFOD2 & $\begin{array}{c}\text { COASY, LMAN2L, } \\
\text { VPSOD1 }\end{array}$ & miR-4792-5p & Gain & $2.00 \mathrm{E}-07$ & $\begin{array}{c}\text { Triglycerides }^{88} \text {, Haemoglobin } \\
\text { leveles, }^{89} \\
\text { Hypercholesterolemia }^{90}\end{array}$ \\
\hline 17 & 37921742 & rs907091 & C & $\mathrm{T}$ & $9.50 \mathrm{E}-05$ & IKZF3 & $R G L 3, D D X 11$ & miR-330-5p & Gain & $3.38 \mathrm{E}-07$ & $\begin{array}{c}\text { Esophageal cancer }^{91}, \text { Allergy }^{92} \\
\text { Primary biliary cirrhosis }\end{array}$ \\
\hline 8 & 11643915 & rs804292 & G & $\mathrm{A}$ & 0.00024 & NEIL2 & ZNF583 & miR-143-3p & Loss & $2.00 \mathrm{E}-06$ & Alcohol/nicotine dependence ${ }^{94}$ \\
\hline
\end{tabular}




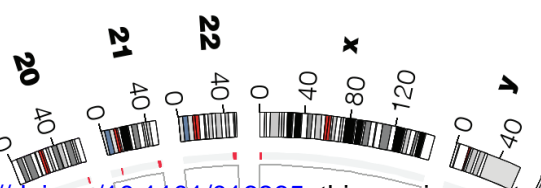

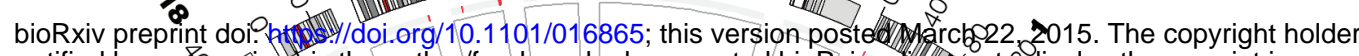
certified by peerorew is the author/funder, who has granted bioRxiv a tere todisplay the preprint in perpe

$>10$

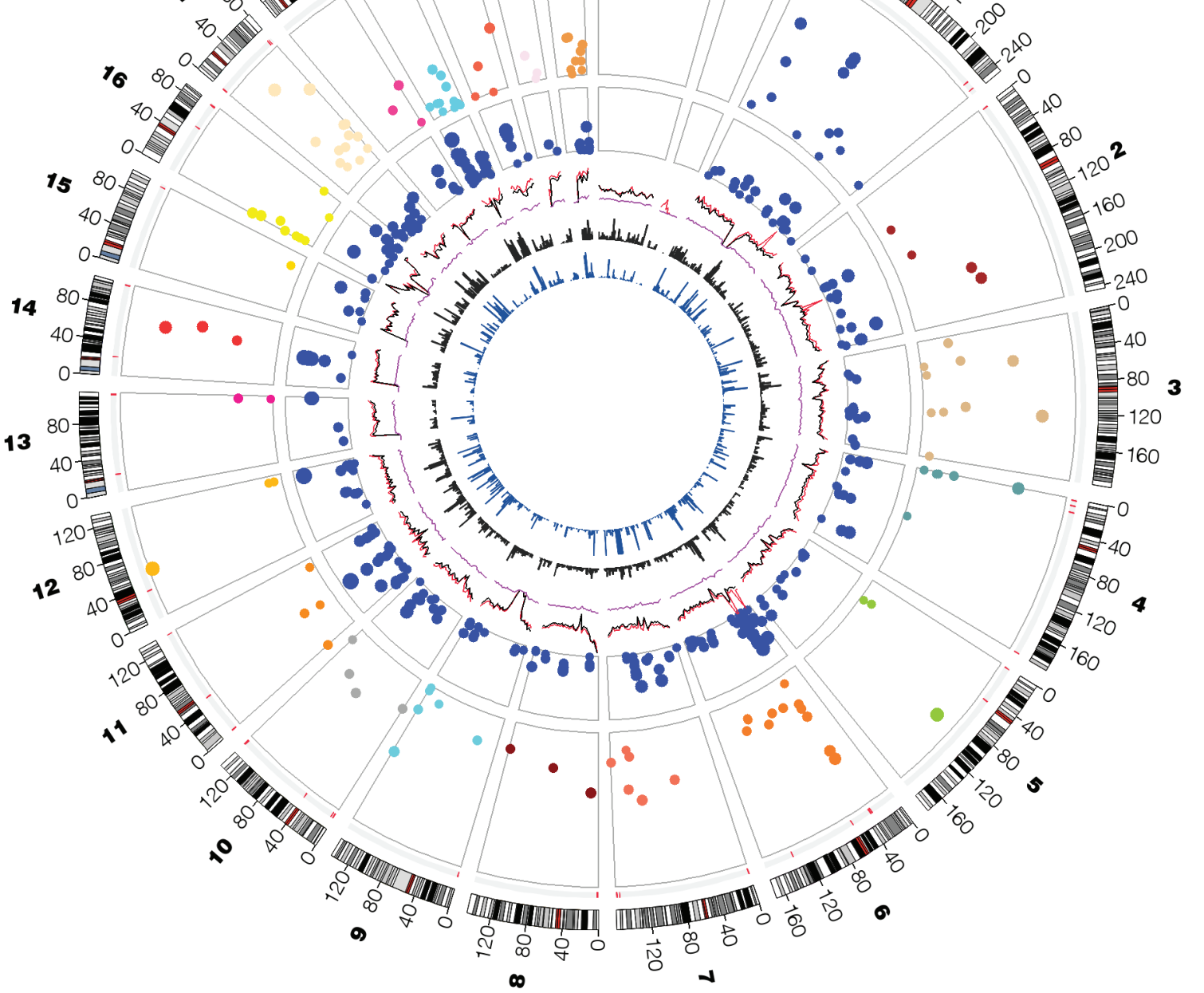


$\mathrm{F} 2$

A bioRxiv preprint doi: https://doi.org/1 $1{ }^{11} 18 d \% 016865$; this version posted $\mathrm{N}$

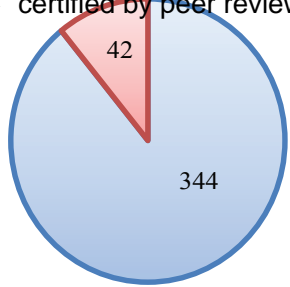

$\square \mathrm{SNV} \square$ Indel

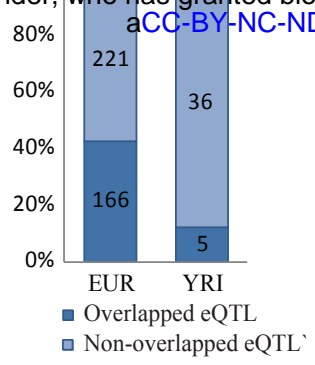

C

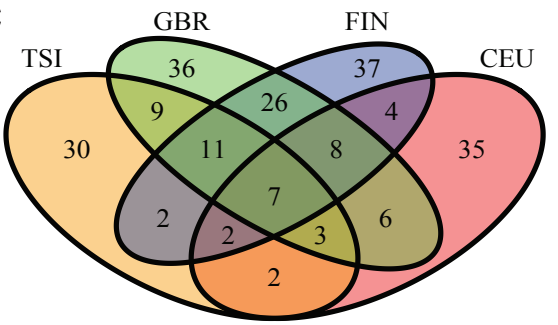

D

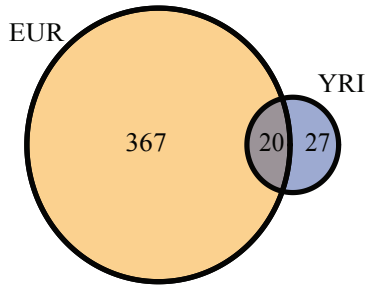


F3 bioRxiv preprint doi: https://doi.org/10.1101/016865; this version posted March 22, 2015. The copyright holder for this preprint (which was not

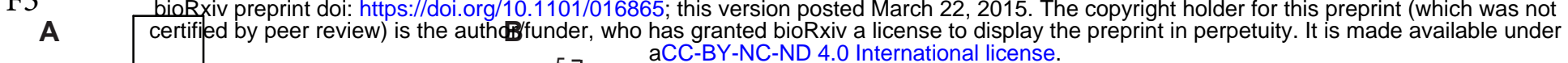
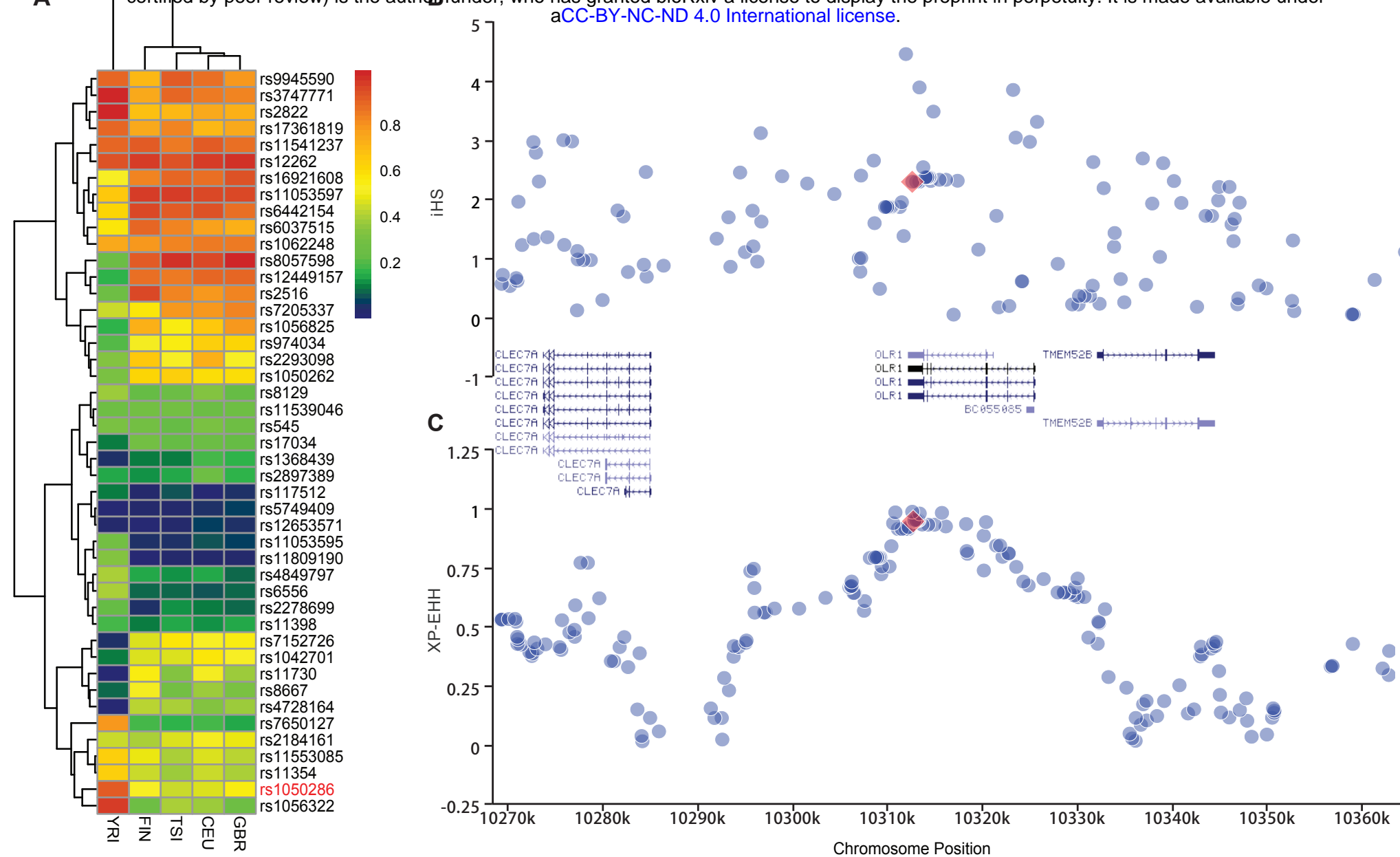
Chromosome Position 
A

A

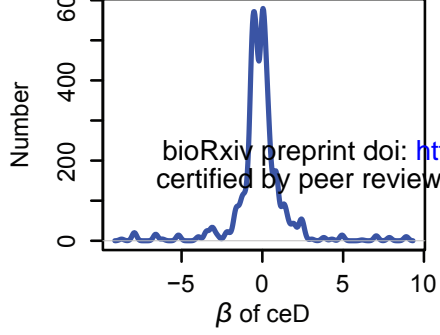

B

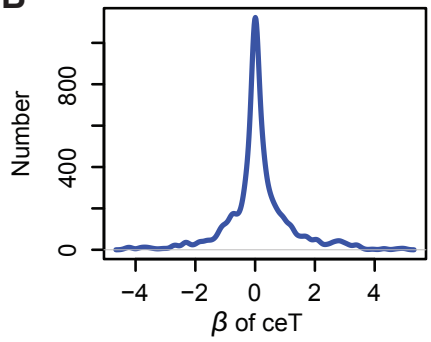

\section{$100 \%$
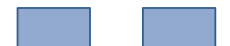 \\ 462}

$8.8 \% / 10.8781 / 016865 ;$ this version poste certified by peer review) is the author/funder, wha twas granted bioRxiv

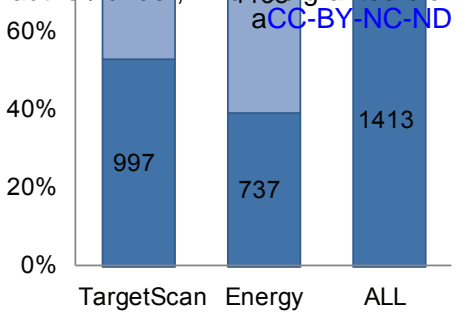

- Concordant with funtional prediction

$\square$ Non-concordant unit 
A

Allele: A

Y hsa-miR-296-5p

bioRxiv preprin MFE:-26.7 kcal/mol certified by peeer review) is the author/fun
B

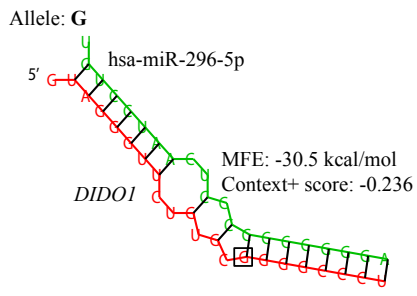

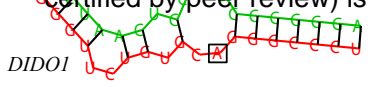

C

rs1056984 - DIDO1
D

rs1056984 - TNFAIP8L1
$\mathbf{F}$

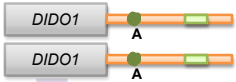
$40-$

$\therefore: \because \because \because: \quad: \because$

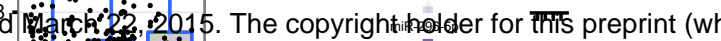
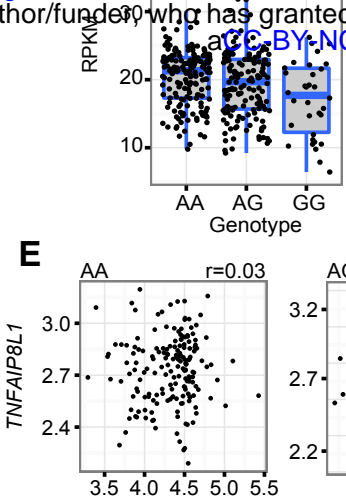

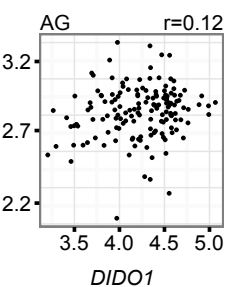

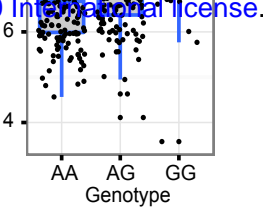

GG

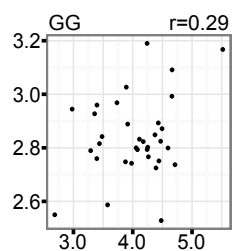
petuity. It is made av \begin{tabular}{|l|l|}
\hline TNFAIP8L1 & TII \\
\hline TNFAIP8L1 & TII \\
\hline
\end{tabular}

rs1056984

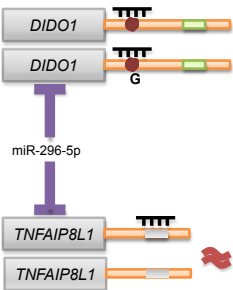


A

\section{AXIN1}

B

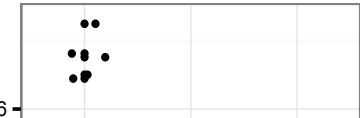

bioRxiv preprint doi: https://doi.arg\$10.1101\%6865; this vers certified by peer review) is the author/funder, who has grantec ABHD\&

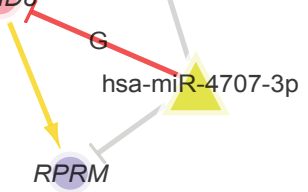

C

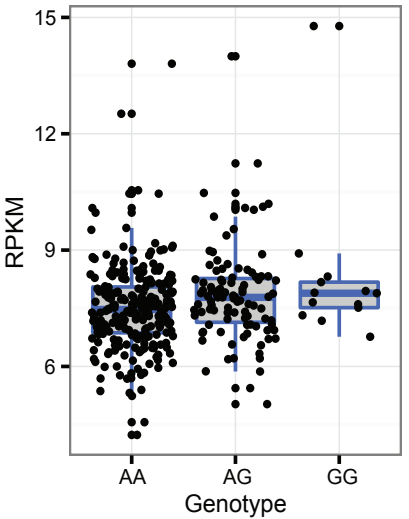

D

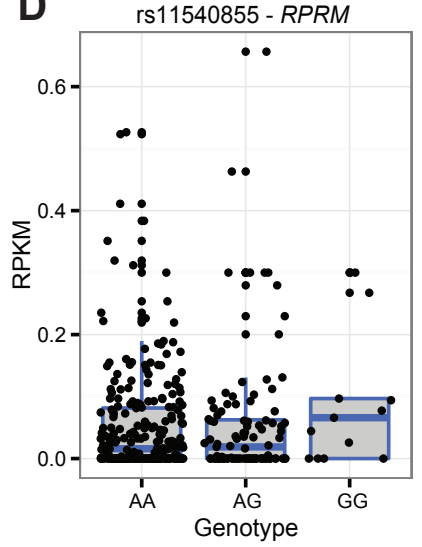

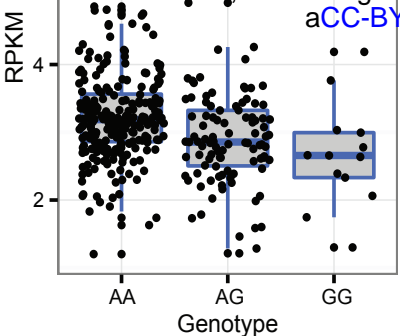
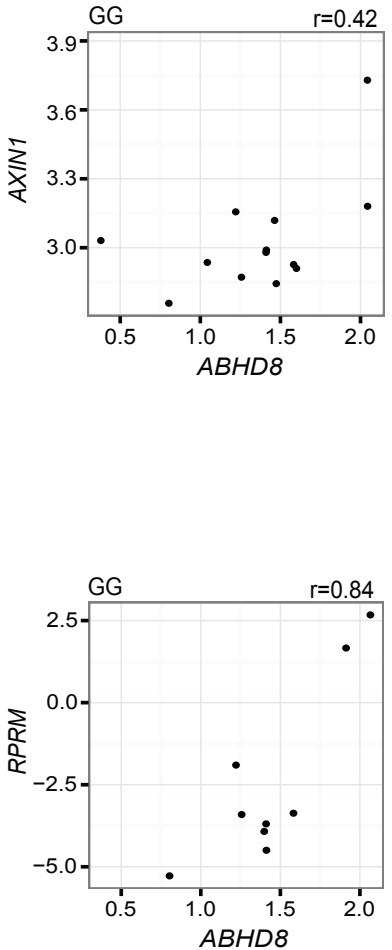